\title{
Genetic characterization of skull base chondrosarcomas
}

\author{
Hiroki Kanamori, MD, ${ }^{1}$ Yohei Kitamura, MD, PhD, ${ }^{1}$ Tokuhiro Kimura, MD, PhD, ${ }^{2}$ \\ Kazunari Yoshida, MD, PhD, ${ }^{1}$ and Hikaru Sasaki, MD, PhD1 \\ Departments of ${ }^{1}$ Neurosurgery and ${ }^{2}$ Pathology, Keio University School of Medicine, Tokyo, Japan
}

\begin{abstract}
OBJECT Although chondrosarcomas rarely arise in the skull base, chondrosarcomas and chordomas are the 2 major malignant bone neoplasms occurring at this location. The distinction of these 2 tumors is important, but this distinction is occasionally problematic because of radiological and histological overlap. Unlike chordoma and extracranial chondrosarcoma, no case series presenting a whole-genome analysis of skull base chondrosarcomas (SBCSs) has been reported. The goal of this study is to clarify the genetic characteristics of SBCSs and contrast them with those of chordomas.
\end{abstract}

METHODS The authors analyzed 7 SBCS specimens for chromosomal copy number alterations (CNAs) using comparative genomic hybridization (CGH). They also examined IDH1 and IDH2 mutations and brachyury expression.

RESULTS In CGH analyses, the authors detected CNAs in 6 of the 7 cases, including chromosomal gains of 8q21.1, 19, 2q22-q32, 5qcen-q14, 8q21-q22, and 15qcen-q14. Mutation of IDH1 was found with a high frequency (5 of 7 cases, $71.4 \%$ ), of which R132S was most frequently mutated. No IDH2 mutations were found, and immunohistochemical staining for brachyury was negative in all cases.

CONCLUSIONS To the best of the authors' knowledge, this is the first whole-genome study of an SBSC case series. Their findings suggest that these tumors are molecularly consistent with a subset of conventional central chondrosarcomas and different from skull base chordomas.

http://thejns.org/doi/abs/10.3171/2014.12.JNS142059

KEY WORDS skull base chondrosarcomas; chordoma; comparative genomic hybridization; IDH1; IDH2; brachyury; oncology

A

LTHOUGH chondrosarcoma is the third most frequent primary malignancy of bone after myeloma and osteosarcoma, ${ }^{7}$ skull base chondrosarcomas (SBCSs) are rare, accounting for only approximately $1 \%$ of all chondrosarcomas. ${ }^{6}$ In France, intracranial chondrosarcomas account for $0.05 \%$ of all brain tumors,$^{21}$ and together with chordoma, they account for $0.09 \%$ of central nervous system tumors in the United States. ${ }^{5}$ However, among malignant bone tumors in the skull base, SBCS and chordoma are the 2 major histological types. Distinction of these 2 tumors is important, because SBCSs of the skull base are reported to show significantly better prognosis than chordomas. ${ }^{1,4}$ Clinicians and pathologists are occasionally confronted by diagnostic problems caused by radiological and histological overlap between SBCSs and chordomas, especially with the "chondroid" subtype of chordomas. Recently, brachyury, a new immunohistochemical marker for chordoma, has been used to help distinguish chordomas from chondrosarcomas in addition to traditional markers, including cytokeratin and epithelial membrane antigen (EMA). ${ }^{18}$

Little is known about the genetic characteristics of SBCSs, because unlike for chordomas and extracranial chondrosarcomas, there have been no reports of whole-genome analysis of a case series of SBCSs to date. An early cytogenetic study of a single case of SBCS was described, ${ }^{8}$ in which no chromosomal abnormality was noted. Recent advances in the molecular pathology of extracranial chondrosarcomas have revealed that conventional chondrosarcomas are divided into central and peripheral subtypes,

ABBREVIATIONS AML = acute myeloid leukemia; $C G H=$ comparative genomic hybridization; $C N A=$ copy number alteration; $D I G=$ digoxigenin; $D O P-P C R=$ degenerate oligonucleotide primed-polymerase chain reaction; EMA = epithelial membrane antigen; PFS = progression-free survival; $S B C S=$ skull base chondrosarcoma.

SUBMITTED October 8, 2014. ACCEPTED December 31, 2014.

INCLUDE WHEN CITING Published online July 10, 2015; DOI: 10.3171/2014.12.JNS142059.

DISCLOSURE This work was financially supported by Japan Society for the Promotion of Science KAKENHI (26861166). The authors report that they have no personal financial, or institutional interest in any of the drugs, materials, or devices described in this article. 
and genetic differences between these 2 subtypes reflect the different pathways of tumorigenesis. ${ }^{27}$ In particular, somatic mutations in the IDHI or IDH2 genes (IDH1/2) have been identified as early events in the development of central chondrosarcomas, whereas such mutations are not detected in peripheral chondrosarcomas. ${ }^{2}$ Clarification of the genetic characteristics of SBCSs will be valuable, because it may provide insights into the tumorigenic pathways of SBCSs and be useful in distinguishing SBCSs and chordomas of the skull base.

In the present study, we analyzed chromosomal copy number alterations (CNAs) in 7 cases of SBCS using comparative genomic hybridization (CGH). To the best of our knowledge, this is the first case series of whole-genome analysis of SBCSs. We also investigated IDHI/2 mutations and brachyury expression in these cases and present the comprehensive genetic characteristics of SBCSs in contrast with those of chordomas.

\section{Methods}

\section{Tumor Samples}

Formalin-fixed, paraffin-embedded tumor samples that were resected from 7 patients with SBCS at Keio University Hospital from 1997 to 2010 were used. Histological diagnosis of chondrosarcomas was achieved using $\mathrm{H} \& \mathrm{E}-$ stained sections. Immunohistochemistry for cytokeratin (AE1/AE3) and EMA were performed for cases in which chordomas were difficult to distinguish, and negativity for these markers confirmed the diagnosis of chondrosarcoma. The tumors were graded (Grades I-III) according to the WHO classification. ${ }^{7}$ For comparison, 10 cases of skull base chordoma resected at our hospital with typical histopathological findings were also analyzed. Clinical follow-up data were available for all patients from whom samples had been collected. This work was approved by the institutional review board of Keio University Hospital.

\section{$\mathrm{CGH}$}

CGH analysis was performed according to a previously described protocol..$^{12}$ Briefly, tumor DNA was extracted from microdissected pieces of formalin-fixed, paraffinembedded tissue, and it was amplified using degenerate oligonucleotide primed-polymerase chain reaction (DOPPCR). During tissue microdissection, consecutive H \& Estained sections were inspected, and nonneoplastic tissue was stringently excluded. Tumor DNA was labeled using DOP-PCR with digoxigenin (DIG)-11-dUTP (Roche); reference DNA was amplified from 50 ng of normal female DNA and labeled with biotin-dUTP (Roche). The probe mixture was denatured and hybridized to normal metaphase spreads (Vysis). After removal of unhybridized probes by washing, the metaphase spread was incubated with fluorescein isothiocyanate-conjugated anti-DIG antibody (Roche) and rhodamine-conjugated avidin (Roche). These preparations were then washed again and counterstained with 4,6-diamino-2-phenylinodole (DAPI) in antifade solution. Red, green, and blue images were acquired and fluorescence intensity ratios along chromosomes were quantitated using a CytoVision analysis system (Applied Imaging).

\section{Direct Sequence of IDH1/2}

Tumor DNA was extracted from microdissected pieces of formalin-fixed, paraffin-embedded tissue as mentioned above. Exon 4 of $I D H 1$ and $I D H 2$, which included codon 132 and 172, respectively, was amplified as described previously. ${ }^{26,28}$ After purification of the PCR products, sequence reactions were performed using a 3130xl Genetic Analyzer (Applied Biosystems).

\section{Immunohistochemistry for MIB-1 and Brachyury}

Immunohistochemistry for MIB-1 and brachyury was performed on paraffin-embedded sections. After antigen retrieval by microwave irradiation, the sections were incubated with mouse monoclonal anti-Ki 67 antibody (MIB-1, 1:200; Dako) or rabbit anti-brachyury antibody (H210, 1:400; Santa Cruz Biotechnology). To quantify MIB-1-positive nuclei, staining indices were analyzed in more than 1000 tumor cells from more than 3 typical tumor areas. Brachyury expression was evaluated by 2 investigators, and samples were considered to be positive for brachyury when the nuclei were stained strongly and diffusely, as described previously. ${ }^{13}$

\section{Statistical Analyses}

The progression-free survival (PFS) was defined from the date of initial surgery until the first day of the treatment for recurrence or the last follow-up date. Statistical analysis was conducted using JMP (version 11.2.0, SAS Institute).

\section{Results \\ Clinical Parameters and MIB-1 Index}

All 7 cases of SBCS were in female patients with a median age of 50 years (range 21-77 years; Table 1). The median follow-up period was 83 months (range 8-132 months). Histologically, all cases were conventional chondrosarcomas (Fig. 1A-C) and graded as Grade I (3 cases) or Grade II (4 cases). The extracellular matrix of the tumor tissue was hyaline (hyaline cartilage-like; Fig. 1B) in 3 cases and mixed hyaline and myxoid (hyaline cartilagelike with degenerative myxoid changes; Fig. 1C) in 4 cases. The median MIB-1 index was $1.2 \%$ (range $0.0 \%-11.3 \%$ ). Recurrence was observed in 5 cases $(71.4 \%)$, and the median time from surgery to recurrence was 32 months (range 21-57 months). Gross-total tumor removal, defined by macroscopically complete resection, was performed in 2 cases (28.6\%); subtotal removal, defined as resection of $>90 \%$ of the tumor, was achieved in 2 cases $(28.6 \%)$; and partial removal, defined as resection of $<90 \%$ of the tumor, was achieved in 3 cases (42.9\%). Postoperative irradiation was performed in 1 case.

\section{CGH}

CNAs were detected in 6 cases $(85.7 \%$; Table 1 and Fig. 2). Gains of $8 \mathrm{q} 21.1$ and 19 were detected in 3 cases, and gains of 2q22-q32, 5qcen-q14, 8q21.2-q22, and 15qcenq14 were detected in 2 cases. Among these, gains on chromosomes $2,5,8$, and 15 were exclusively associated with Grade II tumors. There were no apparent correlations between the type of matrix and CNAs. 


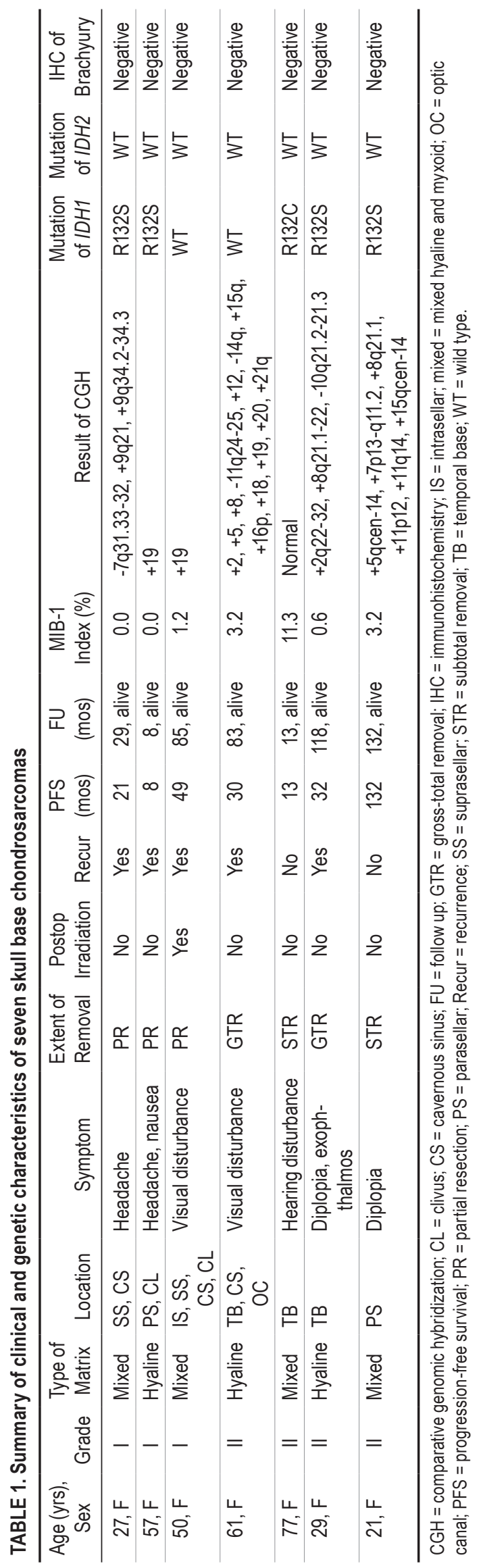

\section{Direct Sequencing of IDH1/2 Mutations}

Mutant IDHI was detected in 5 cases (71.4\%; Table 1, Fig. 3). Among these cases, R132S (C394A) was detected in 4 cases and R132G (C394G) was detected in 1 case. Two cases with wild-type $I D H 1$ also carried wild-type $I D H 2$. In contrast, all 10 cases of skull base chordomas with typical histopathological findings had wild-type IDH1/2.

\section{Immunohistochemistry for Brachyury}

Immunohistochemistry for brachyury was negative in all 7 cases (Table 1, Fig. 1D). In contrast, all 10 chordoma specimens stained positive for brachyury (Fig. 1E).

\section{Statistical Analyses}

The log-rank test demonstrated no significant correlation between PFS and clinical/genetic factors, such as histological grade $(\mathrm{p}=0.309)$, type of extracellular matrix $(p=0.171)$, extent of resection $(p=0.309)$, postoperative irradiation $(p=0.661)$, gain on chromosome $2(p=0.615)$, $5(\mathrm{p}=0.345), 8(\mathrm{p}=0.377), 15(\mathrm{p}=0.345)$, and $19(\mathrm{p}=$ $0.446)$; and mutant IDHI ( $\mathrm{p}=0.986)$.

\section{Discussion}

Several previous studies have reported whole-genome analyses of chondrosarcomas of extracranial sites (common locations) such as the long bones of the extremities, pelvis, and ribs..$^{9,10,15,16,19,23}$ However, to the best of our knowledge, the present study offers the first case series of whole-genome analysis of a rare subset of chondrosarcomas arising in the skull base. The median patient age among our cases was slightly older than that in the largest case study (200 cases) of SBCS (50 vs 39 years, respectively). ${ }^{22}$ The female predominance observed in our series is consistent with a previous report and a recent tumor registry. ${ }^{21,22}$ With respect to the 3 -tiered histological grading of chondrosarcoma, our series included Grade I and II tumors and no Grade III tumors, which is also consistent with a previous study. ${ }^{22}$ When compared with the MIB-1 index for skull base chordomas examined in our previous study, the median MIB-1 index for SBCSs was lower than that for chordomas (1.2\% vs $4.2 \%) .^{14}$

Although results differ greatly among studies, frequent chromosomal alterations in extracranial chondrosarcomas include gains on 2p, 5p, 7, 8q, 14q, 19, 20, and 21q and losses on 4q, 6q, 9p, 13q, and 17p., , $10,15,16,19,23^{2}$ In addition, 8q24.1-qter and 14q24-qter were correlated with shorter overall survival in patients with extracranial chondrosarcomas; ${ }^{15}$ a loss of 6 and gain of 12q12 were associated with higher grade chondrosarcomas; ${ }^{23}$ and losses of $5 \mathrm{q} 14.2$ $\mathrm{q} 21.3,6 \mathrm{q} 16-\mathrm{q} 25.3,9 \mathrm{p} 24.2-\mathrm{p} 12$, and 9p21.3 were associated with higher grade tumors. ${ }^{10}$ In comparison with these data for extracranial cases, our series of SBCSs showed frequent gains of 8q21.1 and 19 in 3 of the 7 cases. Although the association of these CNAs with patients' prognoses was not clear possibly due to the small number of cases, the CNA profile for our SBCS specimens is similar to that for extracranial chondrosarcomas, suggesting the absence of distinct chromosomal alterations specific to the skull base localization of the tumors.

Somatic mutations in IDHI and IDH2 have been rec- 


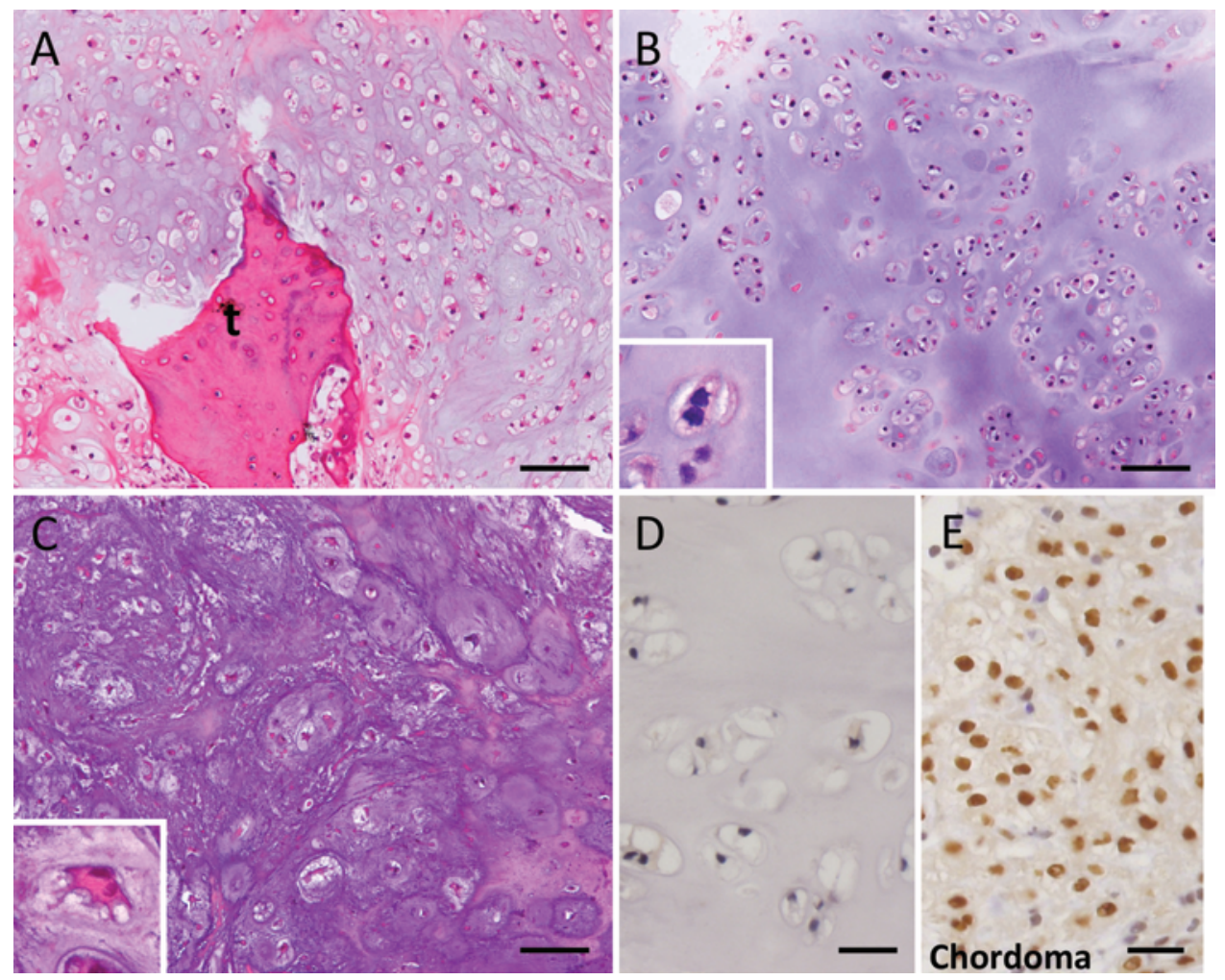

FIG. 1. Histological and immunohistochemical findings of skull base chondrosarcomas in our series. A: The tumors were derived from the proliferation of chondrocyte-like cells with cytological atypia. Permeative or destructive growth is suggested, because preexisting bone trabeculae ( $\mathrm{t}$ ) are trapped within the tumor tissue. B: A case showing predominantly hyaline matrix. The tumor tissue resembles hyaline cartilage, but cellularity is high and the cells show nuclear enlargement and binucleation (inset). C: A case with a mixed hyaline and myxoid matrix. The tumor shows hyaline matrix (right) and degenerative myxoid changes of the matrix (left). Binucleated neoplastic cells are also noted (inset). D and E: Immunohistochemistry for brachyury. Skull base chondrosarcomas were negative for brachyury (D), whereas brachyury staining in chordomas of the skull base was diffusely positive (E). $\mathrm{H}$ \& $E(A-C)$; immunostaining for brachyury $(D$ and $E)$. Bar $=100 \mu \mathrm{m}(A-C) ; 25 \mu \mathrm{m}(D$ and $E)$. Figure is available in color online only.
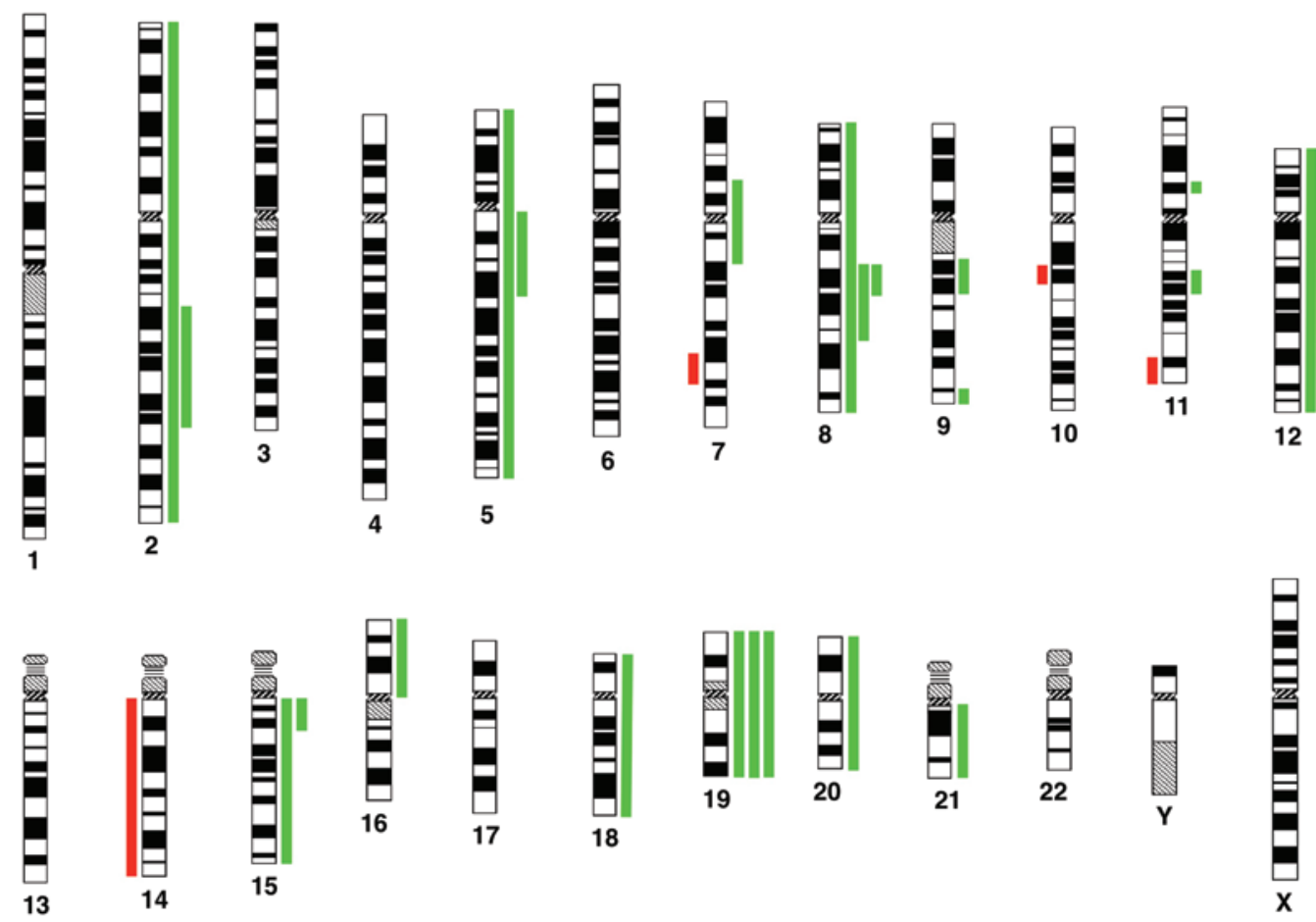

FIG. 2. A summary of the chromosomal aberrations in skull base chondrosarcomas detected by comparative genomic hybridization. Lines to the left of each idiogram represent regions of relative loss; lines to the right represent regions of relative gain. Figure is available in color online only. 

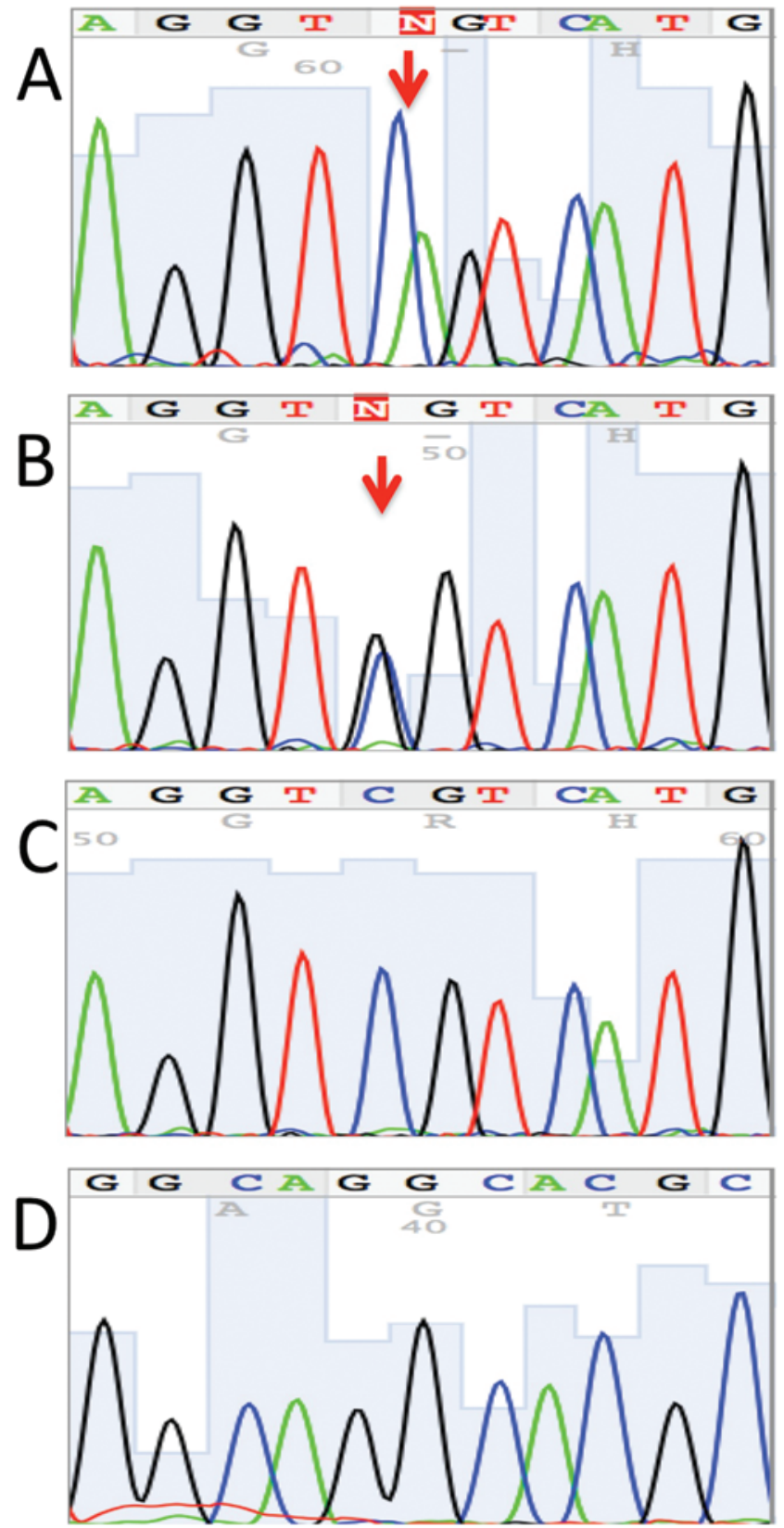

FIG. 3. Representative results of IDH1/2 sequencing. IDH1 mutations R132S (C394A) and R132G (C394G), respectively (A and B). Wild-type $I D H 1$ and $I D H 2$, respectively ( $C$ and $D)$. Figure is available in color online only.

ognized in gliomas and acute myeloid leukemia (AML). Recently, Amary et al. detected mutations in $I D H 1 / 2$ in $56 \%$ of central and periosteal cartilaginous tumors (chondromas and chondrosarcomas) of extracranial sites, but not in peripheral chondrosarcomas. ${ }^{2}$ Subsequently, Arai et al. reported that $I D H 1 / 2$ mutations are also common $(6 / 13$ cases, 46.2\%) in SBCSs but not detected in chordomas of the skull base $\left(0 / 10\right.$ cases). ${ }^{3}$ The present study showed a higher frequency of $I D H I$ mutations (5/7 cases, 71.4\%) than that reported by Arai et al. and confirmed the absence of the mutations in skull base chordomas (0/10 cases). In the present study with skull base bone malignancy, presence of $I D H I$ mutations was associated with chondrosarcoma histopathology rather than chordoma with high sensitivity and specificity ( $71.4 \%$ and $100 \%$, respectively). The most frequent $I D H 1$ mutation detected in our study was R132S (C394A; 4/5 mutations, 80\%), whereas R132C (C394T) was reportedly most common in both the skull base and extracranial chondrosarcomas. ${ }^{2,3,27}$ Both R132S (C394A) and R132C (C394T) occur infrequently in glioma and AML. ${ }^{11,20}$ In fact, R132H (G395A), a preponderant mutation of $I D H 1$ in diffuse gliomas $(92.7 \%)$ and AML $(47 \%),{ }^{11,19}$ was not detected in our study. Because IDH1/2 mutations are considered to play a causal rather than bystander role in tumor development, differences in the sites of these mutations may have a biological significance as well as differences in the cellular origin of the tumors. Although the correlation between mutant IDHI and patients' prognosis was not clear in the present study, further investigation with a large number of cases is warranted.

Because complex chromosomal abnormalities are found in both chondrosarcomas and chordomas, a precise comparison of CNAs appears to be difficult. We previously reported a whole-genome analysis of 37 skull base chordomas using the same methods (DOP-PCR/CGH) applied in the present study, ${ }_{14}$ which enables us to reasonably compare the CGH data for SBCSs and chordomas of the skull base. In our previous report,${ }^{14}$ skull base chordomas frequently showed losses on 1p, 3p, 9, 10,13q, 14q, and 18q and gains on 1q, 2p, 7, and17q, most of which were also reported by other researchers as major abnormalities in chordomas. ${ }^{17,24,25}$ These CNAs were absent or only rarely observed in our SBCS series, implying that the profile of CNAs in SBCS is at least partially different from that in chordomas.

Therefore, the molecular and genetic characteristics of SBCSs in our series, including the pattern of CNAs, the high frequency of $I D H I$ mutations, and the constant negativity of IHC for brachyury, confirm that these tumors are molecularly consistent with a subset of conventional central chondrosarcomas and are in sharp contrast to chordomas, providing further support for distinct tumorigenic pathways for chondrosarcomas and chordomas arising in the bones of the skull base.

\section{Conclusions}

We report the first comprehensive molecular and genetic profile of SBCS. The chromosomal alterations detected in SBCSs via CGH were similar to those reported for extracranial chondrosarcomas. Mutations in IDHI were detected with a higher frequency than that reported previously. To more confidently distinguish SBCSs from chordomas in difficult cases, combined analysis of IDHI/2 sequencing and immunohistochemistry of brachyury may be helpful.

\section{Acknowledgments}

We thank Ms. Naoko Tsuzaki, Ms. Yuko Aikawa, and Ms. Kiyomi Koide from the Department of Neurosurgery at Keio University School of Medicine for their technical assistance.

\section{References}

1. Almefty K, Pravdenkova S, Colli BO, Al-Mefty O, Gokden 
M: Chordoma and chondrosarcoma: similar, but quite different, skull base tumors. Cancer 110:2457-2467, 2007

2. Amary MF, Bacsi K, Maggiani F, Damato S, Halai D, Berisha F, et al: IDH1 and IDH2 mutations are frequent events in central chondrosarcoma and central and periosteal chondromas but not in other mesenchymal tumours. J Pathol 224:334-343, 2011

3. Arai M, Nobusawa S, Ikota H, Takemura S, Nakazato Y: Frequent IDH1/2 mutations in intracranial chondrosarcoma: a possible diagnostic clue for its differentiation from chordoma. Brain Tumor Pathol 29:201-206, 2012

4. Bohman LE, Koch M, Bailey RL, Alonso-Basanta M, Lee JY: Skull base chordoma and chondrosarcoma: influence of clinical and demographic factors on prognosis; a SEER analysis. World Neurosurg 85:806-814, 2014

5. Central Brain Tumor Registry of the United States: CBTRUS Statistical Report: Primary Brain and Central Nervous System Tumors Diagnosed in the United States in 20042007. Hinsdale, IL: Central Brain Tumor Registry of the United States, 2011

6. Dorfman HD, Czerniak B: Bone cancers. Cancer 75 (1 Suppl):203-210, 1995

7. Fletcher CDM, Bridge JA, Hogendoorn P, Mertens F: WHO Classification of Tumours of Soft Tissue and Bone, ed 4. Lyon, France: IARC Press, 2013

8. Gollin SM, Janecka IP: Cytogenetics of cranial base tumors. J Neurooncol 20:241-254, 1994

9. Hallor KH, Staaf J, Bovée JV, Hogendoorn PC, CletonJansen AM, Knuutila S, et al: Genomic profiling of chondrosarcoma: chromosomal patterns in central and peripheral tumors. Clin Cancer Res 15:2685-2694, 2009

10. Hameed M, Ulger C, Yasar D, Limaye N, Kurvathi R, Streck $\mathrm{D}$, et al: Genome profiling of chondrosarcoma using oligonucleotide array-based comparative genomic hybridization. Cancer Genet Cytogenet 192:56-59, 2009

11. Hartmann C, Meyer J, Balss J, Capper D, Mueller W, Christians A, et al: Type and frequency of IDH1 and IDH2 mutations are related to astrocytic and oligodendroglial differentiation and age: a study of 1,010 diffuse gliomas. Acta Neuropathol 118:469-474, 2009

12. Hirose Y, Aldape K, Takahashi M, Berger MS, Feuerstein BG: Tissue microdissection and degenerate oligonucleotide primed-polymerase chain reaction (DOP-PCR) is an effective method to analyze genetic aberrations in invasive tumors. $\mathbf{J}$ Mol Diagn 3:62-67, 2001

13. Jambhekar NA, Rekhi B, Thorat K, Dikshit R, Agrawal M, Puri A: Revisiting chordoma with brachyury, a "new age" marker: analysis of a validation study on 51 cases. Arch Pathol Lab Med 134:1181-1187, 2010

14. Kitamura Y, Sasaki H, Kimura T, Miwa T, Takahashi S, Kawase T, et al: Molecular and clinical risk factors for recurrence of skull base chordomas: gain on chromosome $2 \mathrm{p}$, expression of brachyury, and lack of irradiation negatively correlate with patient prognosis. J Neuropathol Exp Neurol 72:816-823, 2013

15. Larramendy ML, Mandahl N, Mertens F, Blomqvist C, Kivioja $\mathrm{AH}$, Karaharju E, et al: Clinical significance of genetic imbalances revealed by comparative genomic hybridization in chondrosarcomas. Hum Pathol 30:1247-1253, 1999

16. Larramendy ML, Tarkkanen M, Valle J, Kivioja AH, Ervasti $\mathrm{H}$, Karaharju E, et al: Gains, losses, and amplifications of DNA sequences evaluated by comparative genomic hybridization in chondrosarcomas. Am J Pathol 150:685-691, 1997

17. Le LP, Nielsen GP, Rosenberg AE, Thomas D, Batten JM, Deshpande V, et al: Recurrent chromosomal copy number alterations in sporadic chordomas. PLoS ONE 6:e18846, 2011
18. Oakley GJ, Fuhrer K, Seethala RR: Brachyury, SOX-9, and podoplanin, new markers in the skull base chordoma vs chondrosarcoma differential: a tissue microarray-based comparative analysis. Mod Pathol 21:1461-1469, 2008

19. Ozaki T, Wai D, Schäfer KL, Lindner N, Böcker W, Winkelmann W, et al: Comparative genomic hybridization in cartilaginous tumors. Anticancer Res 24:1721-1725, 2004

20. Paschka P, Schlenk RF, Gaidzik VI, Habdank M, Krönke J, Bullinger L, et al: IDH1 and IDH2 mutations are frequent genetic alterations in acute myeloid leukemia and confer adverse prognosis in cytogenetically normal acute myeloid leukemia with NPM1 mutation without FLT3 internal tandem duplication. J Clin Oncol 28:3636-3643, 2010

21. Rigau V, Zouaoui S, Mathieu-Daudé H, Darlix A, Maran A, Trétarre B, et al: French brain tumor database: 5-year histological results on 25756 cases. Brain Pathol 21:633-644, 2011

22. Rosenberg AE, Nielsen GP, Keel SB, Renard LG, Fitzek MM, Munzenrider JE, et al: Chondrosarcoma of the base of the skull: a clinicopathologic study of 200 cases with emphasis on its distinction from chordoma. Am J Surg Pathol 23:1370-1378, 1999

23. Rozeman LB, Szuhai K, Schrage YM, Rosenberg C, Tanke $\mathrm{HJ}$, Taminiau AH, et al: Array-comparative genomic hybridization of central chondrosarcoma: identification of ribosomal protein S6 and cyclin-dependent kinase 4 as candidate target genes for genomic aberrations. Cancer 107:380-388, 2006

24. Scheil S, Brüderlein S, Liehr T, Starke H, Herms J, Schulte $\mathrm{M}$, et al: Genome-wide analysis of sixteen chordomas by comparative genomic hybridization and cytogenetics of the first human chordoma cell line, U-CH1. Genes Chromosomes Cancer 32:203-211, 2001

25. Scheil-Bertram S, Kappler R, von Baer A, Hartwig E, Sarkar M, Serra M, et al: Molecular profiling of chordoma. Int J Oncol 44:1041-1055, 2014

26. Sonoda Y, Kumabe T, Nakamura T, Saito R, Kanamori M, Yamashita Y, et al: Analysis of IDH1 and IDH2 mutations in Japanese glioma patients. Cancer Sci 100:1996-1998, 2009

27. Szuhai K, Cleton-Jansen AM, Hogendoorn PC, Bovée JV: Molecular pathology and its diagnostic use in bone tumors. Cancer Genet 205:193-204, 2012

28. Xu X, Zhao J, Xu Z, Peng B, Huang Q, Arnold E, et al: Structures of human cytosolic NADP-dependent isocitrate dehydrogenase reveal a novel self-regulatory mechanism of activity. J Biol Chem 279:33946-33957, 2004

\section{Author Contributions}

Conception and design: Kitamura and Sasaki. Acquisition of data: Kitamura, Kanamori, Kimura. Analysis and interpretation of data: Kitamura, Kanamori, Kimura, Sasaki. Drafting the article: Kitamura, Kanamori. Critically revising the article: Kimura, Sasaki. Study supervision: Yoshida, Sasaki.

\section{Supplemental Information \\ Current Affiliation}

Dr. Kimura: Yamaguchi University Graduate School of Medicine, Ube, Yamaguchi, Japan.

\section{Correspondence}

Yohei Kitamura, Department of Neurosurgery, Keio University School of Medicine, 35 Shinanomachi, Shinjuku-ku, Tokyo 1608582, Japan. email: ykita@sc4.so-net.ne.jp. 